\title{
Characterization of mouse brain microRNAs after infection with cyst-forming Toxoplasma gondii
}

\author{
Min-Jun $\mathrm{Xu}^{1{ }^{* *}}$, Dong-Hui Zhou ${ }^{1 \dagger}$, Alasdair J Nisbet ${ }^{2}$, Si-Yang Huang ${ }^{1}$, Yi-Fan Fan ${ }^{1,4}$ and Xing-Quan Zhu ${ }^{1,3^{*}}$
}

\begin{abstract}
Background: The obligate intracellular parasite Toxoplasma gondii can interfere with host cell signaling pathways, alter host defense systems and cell cycle control, and establish a chronic infection in the central nervous system. T. gondii infection may alter the expression profile of host microRNAs (miRNAs) which have key regulatory functions at the post-transcriptional level.
\end{abstract}

Methods: Using high-throughput sequencing and real-time quantitative PCR technology, we compared the miRNA expression profiles of uninfected mouse brains with brains from mice at 14 days and 21 days after infection with cyst-forming T. gondii (Type II).

Results: A total of 51.30 million raw reads were obtained from all samples and 495 (14d infected mouse sample), 511 (14d sham-infected control), 504 (21d infected mouse sample) and 514 (21d sham-infected control) miRNA candidates identified. Among these, 414 miRNAs were consistent across all the studied groups, 17 were specific to the $14 \mathrm{~d}$ infected group and 32 were specific to the 21d infected group. In addition, 9 miRNAs were common to both the 14d- and 21d-infected groups. Enrichment analysis for the targets of these miRNAs showed a high percentage of "protein tag" functions. Immune related targets including chemokines, cytokines, growth factors and interleukins were also found.

Conclusions: These results not only showed that the miRNA expression of the host can be changed by the invasion of cyst-forming T. gondii, but also indicated that the host attempts to respond using two tactics: marking proteins with "protein tags" and adaptation of immune related systems.

Keywords: Toxoplasma gondii, Toxoplasmosis, MicroRNA (miRNA), Mouse, Brain, Host regulation

\section{Background}

The apicomplexan parasite Toxoplasma gondii has a wide range of hosts and infects warm-blooded animals worldwide [1-5]. It is also one of the most prevalent parasitic infections in humans with nearly one-third of the population worldwide estimated to be infected [6]. Although infections in adults are typically subclinical, $T$. gondii could possibly persists for the life of the host and can have severe, or even fatal, consequences in the case of congenital transfer or in immune-compromised individuals [7,8]. During

\footnotetext{
* Correspondence: mjxu@yahoo.cn; xingquanzhu1@hotmail.com

${ }^{\dagger}$ Equal contributors

'State Key Laboratory of Veterinary Etiological Biology, Key Laboratory of Veterinary Parasitology of Gansu Province, Lanzhou Veterinary Research Institute, Chinese Academy of Agricultural Sciences, Lanzhou, Gansu Province 730046, PR China

${ }^{3}$ College of Animal Science and Veterinary Medicine, Heilongjiang Bayi Agricultural University, Daqing, Heilongjiang Province 163319, PR China Full list of author information is available at the end of the article
}

invasion of the host cells, a set of specialized secretory organelles is used by the parasite, with some of the associated invasion factors interfering with host cell signaling pathways and altering the host defense systems and cell cycle control [9-12]. However, the modulatory mechanisms of these factors are still not well understood.

microRNAs (miRNAs) are 18-22 nucleotides (nt), non-coding, small RNA molecules which regulate gene expression at the post-transcriptional level and are now considered as a key mechanism of gene regulation $[13,14]$. miRNAs are essential for parasite invasion, development and the ability to respond to environmental and developmental signals, but they also play a role in host cellular differentiation, fitness and defense mechanisms [15-18]. It has previously been reported that $T$. gondii infection can increase the levels of miRNAs in cultured human foreskin fibroblasts (HFFs), such as

\section{Biomed Central}


miR-17 (approximately 92 times) and miR-106b (approximately 25 times) which were known to play crucial roles in cell regulation and were involved in numerous hyperproliferative diseases [19].

Toxoplasma can establish a chronic infection in the central nervous system, where it changes from fastreplicating tachyzoites to bradyzoites and evades the host immune system [20,21] and it was recently suggested that this type of infection may result in the initiation and progression of brain cancer by modifying the miRNA expression of human brain cells [22].

To investigate the roles of specific miRNAs in key regulatory functions during $T$. gondii infection, we therefore compared the miRNA expression profiles of mouse brains during infection with $T$. gondii.

\section{Methods}

\section{Ethics statement}

All animals were handled in strict accordance with good animal practice according to the Animal Ethics Procedures and Guidelines of the People's Republic of China, and the study was approved by the Animal Ethics Committee of Lanzhou Veterinary Research Institute, Chinese Academy of Agricultural Sciences (Permit No. LVRIAEC2010-006).

\section{Collection of mouse brain tissues}

T. gondii PRU strain (Type II) was kindly provided by Professor Hai-Zhu Zhang of Department of Parasitology, Xinxiang Medical College, Henan, China. Forty SpecificPathogen-Free (SPF) grade female Kunming mice (20 mice for control and treatment group respectively), aged from 6 to 8 weeks old, were purchased from the Lanzhou Institute of Biological Products Laboratory Animal Center.

T. gondii cysts were harvested from the brains of infected mice, and then counted using an optical microscope. For the "infected" group, each mouse was inoculated intra-gastrically with 10 cysts; and for the control group, sterile physiological saline was used for the sham inoculation. At 14 day (d) and $21 \mathrm{~d}$ post infection, mice were euthanized, with the whole brain rapidly collected, washed, and stored at $-80 \mathrm{C}$ as described previously [23].

\section{Total RNA and small RNA isolation}

Total RNA was prepared with TRIzol Reagent according to the manufacturer's protocol (Invitrogen Co. Ltd). For each group, total RNA was extracted from 6 biological replicates and then pooled for small RNA isolation. Small RNA of 20-40 bases was obtained as described previously from $10 \mu \mathrm{g}$ total RNA using a Novex 15\% TBE-Urea gel [24]. The fragments were purified and ligated with 5' and $3^{\prime}$ adaptors (Illumina), and reverse transcribed with an RT-PCR kit. All the gels and kits for small RNA purification and reverse transcription were purchased from Invitrogen Co. Ltd.

\section{High-throughput sequencing and bioinformatic analysis}

Samples were sequenced using a Solexa sequencer at HuaDa Genomic Co. Ltd. After base-calling, adaptors and reads with low quality were removed. The raw data were searched against the Rfam databases (http://rfam.sanger.ac. $\mathrm{uk} /$ ) to identify non-coding RNAs, including rRNA, tRNA, snRNA, and snoRNA. Repetitive sequences were identified using RepeatMasker (http://www.repeatmasker.org). The Mus musculus genome at UCSC (http://hgdownload.cse. ucsc.edu/goldenPath/mm9/bigZips/chromFa.tar.gz) was downloaded and used as a reference genome against which filtered reads were mapped using SOAP [25]. Precursors of miRNAs were predicted with Mfold (http://www.bioinfo. rpi.edu/applications/mfold). The predicted mature miRNAs were further matched with known $T$. gondii miRNAs deposited in the Sanger miRBase (http://www.mirbase.org/) to remove possible $T$. gondii contamination. Only those miRNAs having standard stem-loop structure and exact locations on the $M$. musculus reference genome were retained to form the miRNA expression profile of each sample. The miRNA profiles of controls and treatments were then compared with each other to identify specific miRNAs in each sample. Targets of specific miRNAs were predicted using TargetScan with the mouse genes selected $[26,27]$. Functions of the predicted targets were then analyzed with the Gene Ontology (http://www.geneontology. org/) and DAVID databases (http://david.abcc.ncifcrf.gov/).

\section{Analysis of miRNA expression level}

Stem-loop real-time RT-PCR was performed as described previously [28]. The glyceraldehyde-3-phosphate dehydrogenase (Gapdh) of M. musculus (NM_008084.2) was used as endogenous control of each reaction and all reactions were performed using an ABI PRISM ${ }^{\circ} 7300$ Se- $^{-}$ quence Detection System. All primers were synthesized by Shenggong Co. Ltd., China and all reactions were carried out in triplicate. The primer pairs for Gapdh quantification were: F: 5'-GGCCTCCAAGGAGTAAGAAA-3'; R: 5'-GCCCCTCCTGTTATTATGG-3'. The cycles were performed as follows: $50^{\circ} \mathrm{C}$ and $95 \mathrm{C}$ for $2 \mathrm{~min}$ respectively, followed by 30 cycles of $95^{\circ} \mathrm{C}$ for $15 \mathrm{~s}, 60^{\circ}$ for $32 \mathrm{~s}$, and $72 \mathrm{C}$ for $32 \mathrm{~s}$. The relative quantification of each miRNA was calculated using the equation: $\mathrm{N}=2^{-\Delta \mathrm{Ct}}$, $\Delta \mathrm{Ct}=\mathrm{Ct}_{\text {miRNA }}-\mathrm{Ct}_{\text {control }}[29]$.

\section{Results}

\section{Profile characteristics of short RNAs}

A total of 51.30 million raw reads were obtained from all groups. After removing low quality reads and adaptors, 45.53 million high quality reads remained, including 10.48 and 11.04 million reads for the $14 \mathrm{~d}$ infected 
sample and its sham-infected control respectively; and 10.76 and 13.25 million reads for the $21 \mathrm{~d}$ infected sample and its sham-infected control respectively.

Length distribution analysis showed that reads of 2123nt were the most abundant. Repeat analysis revealed that 97 repeat sequence types (representing 5.84\% $7.61 \%$ of the unique reads) of which 11 (Ambi, tRNA:1, LINE/L1:0, LINE/L1:1, LTR/MaLR:0, snRNA:1, SINE/ B4:1, LTR/MaLR:1, SINE/Alu:1, SINE/B2:0, rRNA:1) were most highly represented with read numbers higher than 1000 (1007-20735 reads). Other non-coding RNAs, including tRNA, rRNA, snRNA and snoRNA, represented a total of $24.43 \%-30.65 \%$ of the unique reads of the 4 samples; rRNA was the predominant non-coding RNA (13.31-19.04\% of the unique reads).

\section{Global view of miRNA profiles}

By mapping against the $M$. musculus genome, we obtained 511 (43 novel + 468 known), $495(36+459), 514(53+$ $461)$ and $504(41+463)$ miRNA candidates, with precursors having standard stem-loop structures, from the total RNA of samples of 14d-control, 14d-infection, 21dcontrol, and 21d-infection, respectively. By matching the miRNA candidates with known $M$. musculus miRNAs deposited in the Sanger miRBase, most of the miRNA candidates were previously known, with only $7.84 \%$ (36/ $459)-11.50 \%$ (53/461) identified as novel.

There were 16 miRNAs with copy numbers higher than 100,000 , ranging from 121,582 to $2,233,052$ copies. These 16 miRNAs belonged to 7 families; let-7, mir-101, mir-128, mir-107, mir-140, mir-29a and mir-124. The let-7 family, which was the most highly represented in each miRNA profile, had 8 members present from mmu-let-7a to mmu-let-7i. For the mir-101 and mir-128 families, two members were identified in each; mir-101a and mir-101b, and mir-128-1 and mir-128-2.

\section{Comparison of miRNA profiles}

414 miRNAs were common amongst the infected and sham-infected (control) groups (Figure 1). Compared with controls, 45 different miRNAs occurred in the 14dinfected sample (Figure 1a, b, c, e); and 59 different miRNAs occurred in 21d-infected sample (Figure 1d, e, $\mathrm{f}, \mathrm{g}$ ). Among these, 9 miRNAs (Figure 1e) were shared between the two "infected" groups, representing miRNAs, which persist after T. gondii infection. Moreover, 17 miRNAs (Figure 1a) were specific to the $14 \mathrm{~d}$-infected sample and 32 miRNAs (Figure 1d) were specific to the 21d-infected sample.

\section{Target prediction and functional analysis}

The 17 and 32 specific miRNAs from $14 \mathrm{~d}$ - and 21dinfected samples, as well as the 9 miRNAs common to both time points post-infection, were used for target

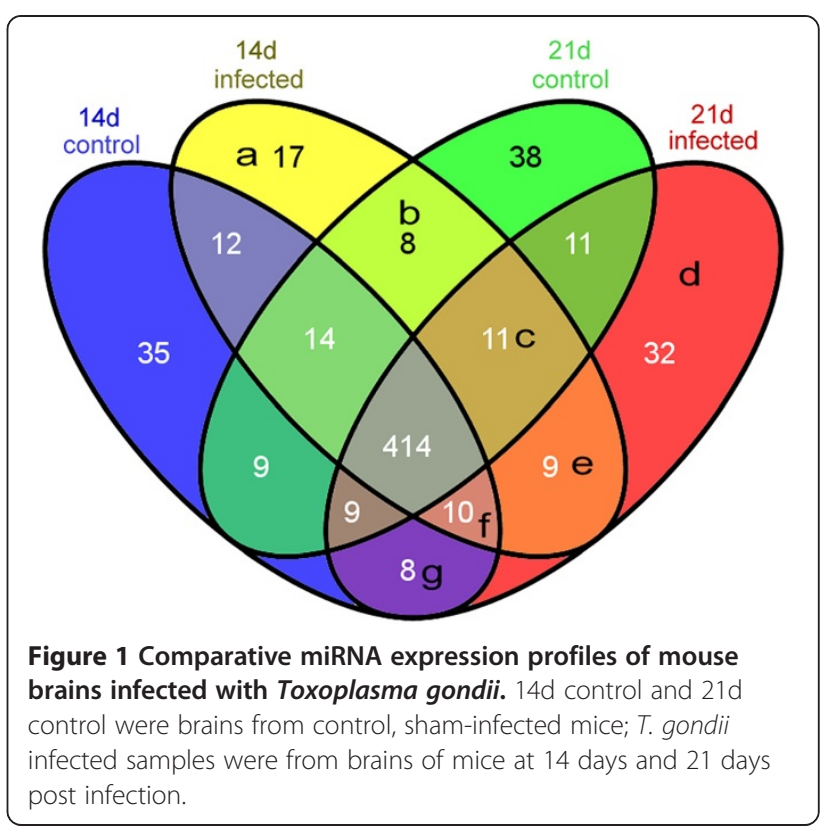

prediction (Additional file 1: Table S1). For the 17 specific miRNAs of the $14 \mathrm{~d}$-infected sample, 1954 targets were found in total, with 115 on average; the miRNA that possessed the highest number of targets was mmu-miR-9 (1076 targets), with the best matched target being KEL (Kell blood group, metallo-endopeptidase, NM_000420). For the 32 specific miRNAs of the 21d-infected sample, 2407 targets were found in total with 78 on average; the miRNA that possessed the highest number of targets was mmu-miR-694 (888 targets) with the best matched target being CHCHD1 (coiled-coil-helix-coiled-coil-helix domain containing 1, NM_203298). For the 9 miRNAs common to both time points post-infection, the total number of targets was 501 with 63 on average. The miRNA that had the highest number of predicted targets was mmu-miR-5107 (212 targets) and the best matched target was Pafah2 (platelet-activating factor acetylhydrolase 2, NM_133880).

For global analysis, the GO items of the targets were enrichment-analyzed (Figure 2). For the 9 miRNAs common to both time points post-infection, the numbers of different GO items represented were generally lower than those of the 14d-specific and 21d-specific miRNAs at all of the 3 classifications in GO analysis (cellular component, molecular function and biological process). For cellular component, only "synapse" had a marginally higher percentage in the common miRNAs than in the $14 \mathrm{~d}$ - or $21 \mathrm{~d}$-specific miRNAs (Figure 2, annotated with "1"), however, there was a significantly higher percentage of the common, rather than the post-infection time point specific miRNAs focused on the "protein tag" function (Figure 2, annotated with " 8 "). In addition, a further two functions ("auxiliary transport protein" and "molecular 


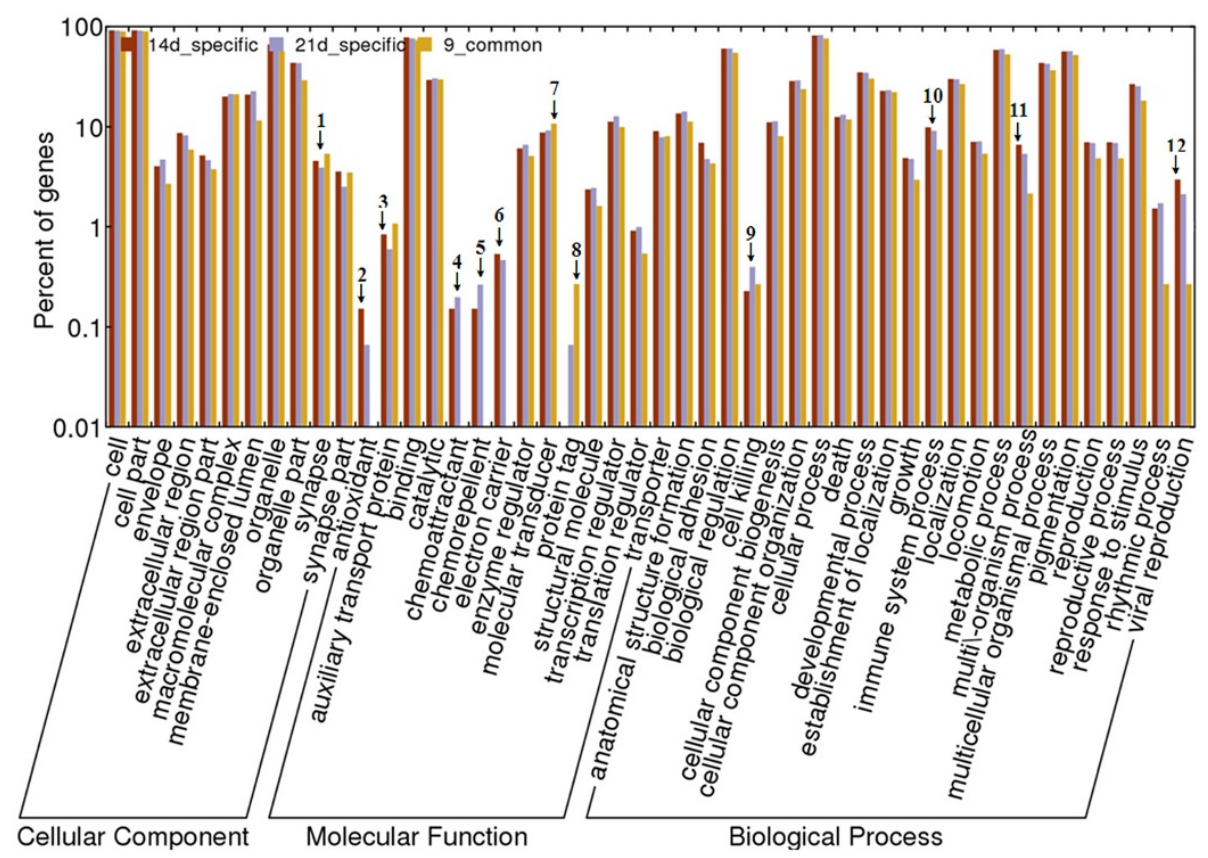

Figure 2 Enrichment analysis for the targets of miRNAs in the brains of Toxoplasma gondii-infected mice 14 and 21 days postinfection. Annotated numbers were used to mark columns that had different percentages in different samples.

transducer") were also represented marginally higher in the common, rather than the post-infection time point specific miRNAs (Figure 2, annotated with " 3 " and "7").

Targets of the $14 \mathrm{~d}$-specific and $21 \mathrm{~d}$-specific miRNAs contained 4 functions, which were not identified in the 9 common miRNAs; "antioxidant", "chemoattractant", "chemorepellent" and "electron carrier" (Figure 2, annotated with "2", "4", "5", and "6"). Targets of the 14dspecific miRNAs had significantly higher representation in the "antioxidant" function than those of the 21d-specific miRNAs (Figure 2, annotated with "2") and marginally higher representation in the "electron carrier" function (Figure 2, annotated with "6"). Targets of the 21d-specific miRNAs were relatively more abundant in the "chemoattractant" and "chemorepellent" functions (Figure 2, annotated with "4" and "5"), and "protein tag" functions (Figure 2, annotated with " 8 "). For biological process, the targets of 14d-specific miRNAs had marginally higher percentages in "immune system process", "multi-organismal process" and "viral reproduction" processes than either the common miRNAs or the 21d-infected miRNAs (Figure 2, annotated with "10", "11" and "12"), but targets of the 21dspecific miRNAs had significantly higher representation in the "cell killing" process (Figure 2, annotated with "9").

Among the targets, some immune-related functionality was found, including "Chemokine", "Cytokine", "Growth factor" and "Interleukin". For chemokine-related targets/ factors/receptors, there were 12 targets associated with $14 \mathrm{~d}$-specific miRNAs, including "binding activity"
(GO:0031726) and "antagonist activity" (GO:0046817), but only one target (GO:0008009) associated with 21d-specific miRNAs and the 9 miRNAs common to both time points post-infection. For cytokine-related factors, 3 and 9 targets were associated with the 9 miRNAs common to both time points post-infection and 14d-specific miRNAs respectively, while there were 18 targets associated with the $21 \mathrm{~d}$ specific miRNAs. For "growth factor", there were 12, 57 and 66 targets respectively for the 9 miRNAs common to both time points post-infection, 14d-specific and 21dspecific miRNAs; including fibroblast growth factor (GO:0005105), epidermal growth factor (GO:0005154), nerve growth factor (GO:0048406) and vascular endothelial growth factor (GO:0005021). For "interleukins" (IL), receptors of IL-3, IL-6, and IL-23 were amongst the targets of the 9 miRNAs common to both time points postinfection; receptors of IL-1 and IL-7 were associated with the $14 \mathrm{~d}$-specific miRNAs and the majority of the interleukin receptors were amongst the targets of the 21d-specific miRNAs, including IL-1, IL-2, IL-3, IL-7 and IL-23.

\section{miRNA quantification}

Six representative miRNAs, including mmu-mir-5107 and mmu-miR-novel-1 (common to both time points postinfection); 14df-m0014-5p and 14df-m0027-3p (14d-specific miRNAs), and 21df-m0027-5p and 21df-m0013-5p (21d-specific miRNAs), which had higher copy numbers than others, were chosen and used in a quantitative PCR analysis (Figure 3). All 6 miRNAs were successfully 


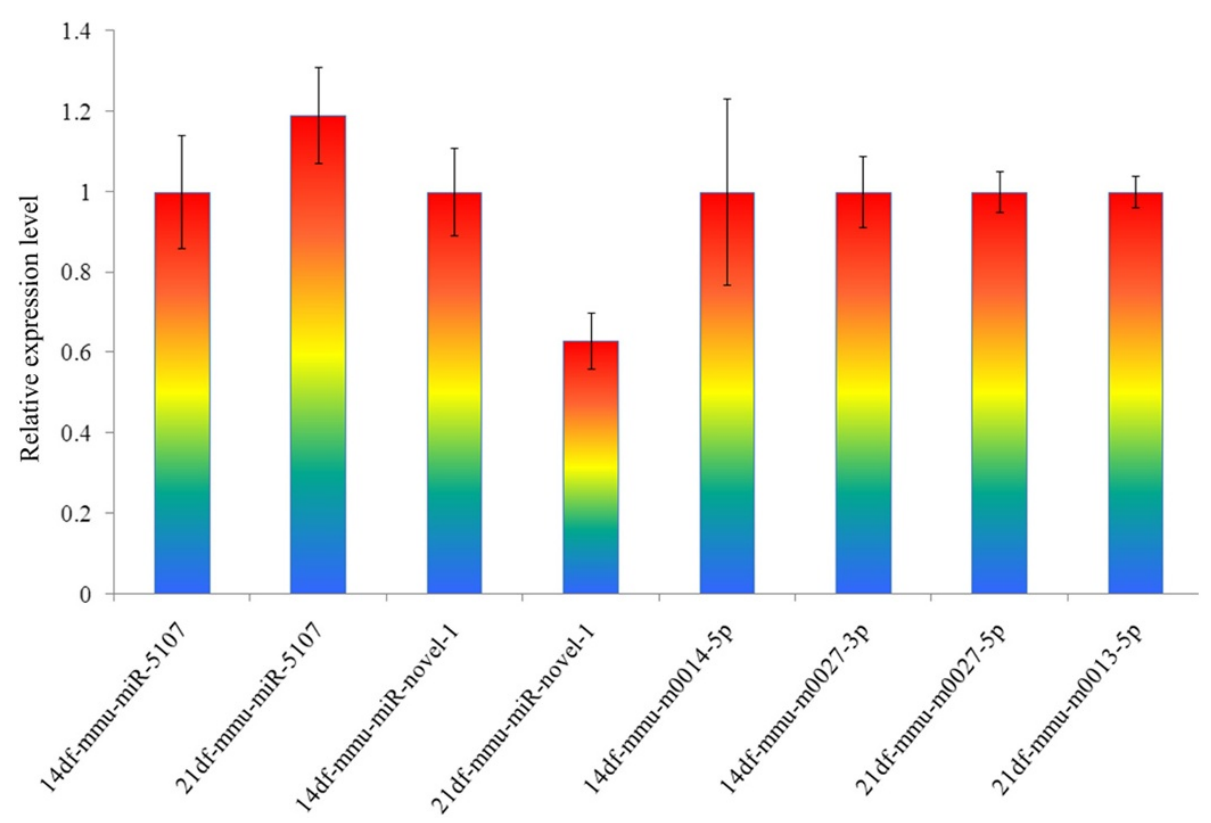

Figure 3 Quantitative PCR analysis of the 6 representative miRNAs.

amplified. The mmu-miR-5107 was expressed marginally higher in the $21 \mathrm{~d}$ sample $(1.19 \pm 0.12)$ than in the $14 \mathrm{~d}(1 \pm$ 0.14 ), while the expression level of mmu-miR-novel-1 was slightly lower in the $21 \mathrm{~d}$ sample $(0.63 \pm 0.07)$ than that in the $14 \mathrm{~d}$ RNA $(1 \pm 0.11)$. The other 4 miRNAs were at nearly same level as the endogenous control with expression levels of $1 \pm 0.23$ (14df-mmu-m0014-5p), $1 \pm 0.09$ (14df-mmu-m0027-3p), $1 \pm 0.05$ (21df-mmu-m0027-5p), and $1 \pm 0.04$ (21df-mmu-m0013-5p).

\section{Discussion}

Invasion and survival of parasitic protozoa in a hostile host environment requires multiple gene expression adaptations for modifying or manipulating the host environment, some of which are thought to involve parasite-derived miRNAs [30]. In addition, host-derived miRNAs control host protein production; for example human miRNAs are reported to control the expression of around $60 \%$ of human protein-coding genes and, because of these key regulatory functions, deregulation of miRNAs has been associated with the pathogenesis, progression and prognosis of different diseases [31]. To analyze the interplay between parasite and host at the miRNA levels, we investigated the changes in miRNA profiles of mouse brains 14 and $21 \mathrm{~d}$ after infection with cyst-forming $T$. gondii, which had more cyst burdens than other time points [32].

Total numbers of miRNAs identified were similar $(\sim 500)$ in infected brain samples and controls, and most of these (414) were conserved in both infected and uninfected brain samples, regardless of the time points post infection, indicating fundamental regulatory functions of most of these miRNAs. In addition, multiple time and infection status-specific miRNAs were also found indicating that the miRNA expression profiles changed over time, whether parasites were present or not. For the 14d sham-infected control, there were 35 specific miRNAs which were not detected following $T$. gondii invasion but were replaced by the 17 "14d infection-specific" miRNAs. In a recent study [19], it was found that $T$. gondii infection changed the expression level of miR-17 (approximately 92 times) and miR-106b (approximately 25 times) in cultured HFFs, although the mechanisms driving their altered expression are unknown. These miRNAs were supposed to regulate development and metabolism of host cells. During the invasion of host cells by the parasite, some metabolism pathways were closed and redirected, and new pathways conducted by the parasite were generated as well, therefore, old miRNAs were replaced by new ones. A similar phenomenon was also found in the mouse brains at 21d post-infection in the present study. Therefore, $T$. gondii invasion altered the host miRNA expression, in agreement with the results of Zeiner et al. [19], although there were many factors, which are difficult to be well controlled, such as the host condition, different stress factors and the virulence of the parasite, which may influence the results.

The 21d infected sample contained miRNAs whose predicted targets possessed "protein tag" functions - a function which was absent from the targets of the miRNAs derived from the 14d-specific sample (Figure 2). In addition "protein tag" targets were also found in the 9 
miRNAs common to both time points post-infection. This indicates that miRNAs for which targets have "protein tag" functions were present after 14d post infection and further enhanced by $21 \mathrm{~d}$ post infection. Toxoplasma gondii can change host metabolism and reproduce itself alongside host processes and factors $[9,12]$, therefore, correct protein tags are required for the host to identify "self" proteins and guide them into the appropriate metabolic pathways, which may be a competitive mechanism for dealing with parasitism. In connection with this, it has been reported that the secreted proteins of the parasite may be undergoing accelerated evolution, possibly as a response to stronger selective pressure from host [30]. The "protein tag" function of the host miRNA targets may thus represent a host anti-invasion strategy in response to such accelerated evolution of the parasite.

Immune-related targets including chemokines, cytokines, growth factors and interleukins were also found. These factors all have roles in the host immune response to parasite challenge: chemokines and their receptors are important in the control of parasite replication and acute cardiac inflammation, and trigger proinflammatory responses to many microbial pathogens [33,34]. At the time point of $14 \mathrm{~d}$ post infection more chemokine targets were identified than at $21 \mathrm{~d}$ post infection, with 12 chemokine-related targets in the $14 \mathrm{~d}$ sample and only 1 in the $21 \mathrm{~d}$ sample. In contrast, the numbers of cytokine, growth factor and interleukin targets were much higher in the $21 \mathrm{~d}$ sample than in the 14d sample. Interleukins have been reported as being important host protection factors against parasites, such as gastrointestinal nematodes and protozoan parasites $[35,36]$ and, from the data presented here, the roles of interleukin targets as well as those for cytokines and growth factors increase with the development of $T$. gondii infections.

\section{Conclusions}

The present study characterized the expression profiles of miRNA in the brain of mice during the invasion of $T$. gondii. This analysis showed that the miRNA expression of the host brain can be altered by the invasion of cystforming $T$. gondii, and that the host might attempt to protect itself by marking proteins with "protein tags" and through the increased regulation of immune related systems.

\section{Additional file}

Additional file 1: Table S1. Common and specific miRNAs of

Toxoplasma gondii infected mouse brain at $14 \mathrm{~d}$ and $21 \mathrm{~d}$ post infection.

\section{Competing interests}

The authors declare that they have no competing interests.

\section{Authors' contributions}

$X Q Z$ conceived and designed the study, and critically revised the manuscript. MJX and DHZ performed the experiments, analyzed the data and drafted the manuscript. AJN, SYH and YFF helped in study design, study implementation and manuscript revision. All authors read and approved the final manuscript.

\section{Acknowledgments}

Project support was provided by the National Natural Science Foundation of China (Grant Nos. 31230073 and 31172316) to XQZ.

\section{Author details}

${ }^{1}$ State Key Laboratory of Veterinary Etiological Biology, Key Laboratory of Veterinary Parasitology of Gansu Province, Lanzhou Veterinary Research Institute, Chinese Academy of Agricultural Sciences, Lanzhou, Gansu Province 730046, PR China. ${ }^{2}$ Vaccines and Diagnostics, Moredun Research Institute, Pentlands Science Park, Midlothian, Scotland EH26 OPZ, UK. ${ }^{3}$ College of Animal Science and Veterinary Medicine, Heilongjiang Bayi Agricultural University, Daqing, Heilongjiang Province 163319, PR China. ${ }^{4}$ College of Veterinary Medicine, Sichuan Agricultural University, Ya'an, Sichuan Province 625014, PR China.

Received: 16 April 2013 Accepted: 19 May 2013

Published: 29 May 2013

\section{References}

1. Dubey JP: Toxoplasmosis of Animals and Humans. Boca Raton, Florida: CRC Press; 2010:313.

2. Nardoni S, Angelici MC, Mugnaini L, Mancianti F: Prevalence of Toxoplasma gondii infection in Myocastor coypus in a protected Italian wetland. Parasit Vectors 2011, 4:240.

3. Chen J, Xu MJ, Zhou DH, Song HQ, Wang CR, Zhu XQ: Canine and feline parasitic zoonoses in China. Parasit Vectors 2012, 5:152.

4. Tian YM, Dai FY, Huang SY, Deng ZH, Duan G, Zhou DH, Yang JF, Weng YB, Zhu XQ, Zou FC: First report of Toxoplasma gondii seroprevalence in peafowls in Yunnan Province. Southwestern China. Parasit Vectors 2012, 5:205

5. Zhou P, Chen Z, Li HL, Zheng H, He S, Lin RQ, Zhu XQ: Toxoplasma gondii infection in humans in China. Parasit Vectors 2011, 4:165.

6. Montoya JG, Liesenfeld O: Toxoplasmosis. Lancet 2004, 363:1965-1976.

7. Sibley LD, Boothroyd JC: Virulent strains of Toxoplasma gondii comprise a single clonal lineage. Nature 1992, 359:82-85.

8. Olguin-Lamas A, Madec E, Hovasse A, Werkmeister E, Callebaut I, Slomianny C, Delhaye S, Mouveaux T, Schaeffer-Reiss C, Van Dorsselaer A, Tomavo S: A novel Toxoplasma gondii nuclear factor TgNF3 is a dynamic chromatinassociated component, modulator of nucleolar architecture and parasite virulence. PLoS Pathog 2011, 7:e1001328.

9. Boothroyd JC, Dubremetz JF: Kiss and spit: the dual roles of Toxoplasma rhoptries. Nat Rev Microbiol 2008, 6:79-88.

10. Saeij JP, Boyle JP, Coller S, Taylor S, Sibley LD, Brooke-Powell ET, Ajioka JW, Boothroyd JC: Polymorphic secreted kinases are key virulence factors in toxoplasmosis. Science 2006, 314:1780-1783.

11. Saeij JP, Coller S, Boyle JP, Jerome ME, White MW, Boothroyd JC: Toxoplasma co-opts host gene expression by injection of a polymorphic kinase homologue. Nature 2007, 445:324-327.

12. Molestina RE, El-Guendy N, Sinai AP: Infection with Toxoplasma gondii results in dysregulation of the host cell cycle. Cell Microbiol 2008, 10:1153-1165.

13. Du T, Zamore PD: Beginning to understand microRNA function. Cell Res 2007, 17:661-663.

14. Lim LP, Glasner ME, Yekta S, Burge CB, Bartel DP: Vertebrate microRNA genes. Science 2003, 299:1540.

15. Liu Q, Tuo W, Gao H, Zhu XQ: MicroRNAs of parasites: current status and future perspectives. Parasitol Res 2010, 107:501-507.

16. Lin WC, Li SC, Lin WC, Shin JW, Hu SN, Yu XM, Huang TY, Chen SC, Chen HC, Chen SJ, Huang PJ, Gan RR, Chiu CH, Tang P: Identification of microRNA in the protist Trichomonas vaginalis. Genomics 2009 93:487-493.

17. TenOever BR: RNA viruses and the host microRNA machinery. Nat Rev Microbiol 2013, 11:169-180.

18. Zhai H, Fesler A, Ju J: MicroRNA: a third dimension in autophagy. Cell Cycle 2013, 12:246-250. 
19. Zeiner GM, Norman KL, Thomson JM, Hammond SM, Boothroyd JC: Toxoplasma gondii infection specifically increases the levels of key host microRNAs. PLoS One 2010, 5:e8742.

20. Weiss LM, Kim K: The development and biology of bradyzoites of Toxoplasma gondii. Front Biosci 2000, 5:D391-D405.

21. Bohne W, Holpert M, Gross U: Stage differentiation of the protozoan parasite Toxoplasma gondii. Immunobiology 1999, 201:248-254.

22. Thirugnanam S, Rout N, Gnanasekar M: Possible role of Toxoplasma gondii in brain cancer through modulation of host microRNAs. Infect Agent Cancer 2013, 8:8.

23. Zhou DH, Zhao FR, Huang SY, XU MJ, Song HQ, Su C, Zhu XQ: Changes in the proteomic profiles of mouse brain after infection with cyst-forming Toxoplasma gondii. Parasit Vectors 2013, 6:96.

24. Xu MJ, Liu Q, Nisbet AJ, Cai XQ, Yan C, Lin RQ, Yuan ZG, Song HQ, He XH, Zhu XQ: Identification and characterization of microRNAs in Clonorchis sinensis of human health significance. BMC Genomics 2010, 11:521.

25. Li R, YU C, Li Y, Lam TW, Yiu SM, Kristiansen K, Wang J: SOAP2: an improved ultrafast tool for short read alignment. Bioinformatics 2009, 25:1966-1967.

26. Lewis $B P$, Burge $C B$, Bartel DP: Conserved seed pairing, often flanked by adenosines, indicates that thousands of human genes are microRNA targets. Cell 2005, 120:15-20.

27. Grimson A, Farh KK, Johnston WK, Garrett-Engele P, Lim LP, Bartel DP: MicroRNA targeting specificity in mammals: determinants beyond seed pairing. Mol Cell 2007, 27:91-105.

28. Chen C, Ridzon DA, Broomer AJ, Zhou Z, Lee DH, Nguyen JT, Barbisin M, Xu NL, Mahuvakar VR, Andersen MR, Lao KQ, Livak KJ, Guegler KJ: Real-time quantification of microRNAs by stem-loop RT-PCR. Nucleic Acids Res 2005, 33:e179.

29. Livak KJ, Schmittgen TD: Analysis of relative gene expression data using real-time quantitative PCR and the 2(-Delta Delta $C(T))$ Method. Methods 2001, 25:402-408.

30. Harcus YM, Parkinson J, Fernandez C, Daub J, Selkirk ME, Blaxter ML, Maizels RM: Signal sequence analysis of expressed sequence tags from the nematode Nippostrongylus brasiliensis and the evolution of secreted proteins in parasites. Genome Biol 2004, 5:R39.

31. Akbari MF, Pieters $R$, den Boer ML: The hunting of targets: challenge in miRNA research. Leukemia 2013, 27:16-23.

32. Djurkovic-Djakovic O, Djokic V, Vujanic M, Zivkovic T, Bobic B, Nikolic A, Slavic K, Klun I, Ivovic V: Kinetics of parasite burdens in blood and tissues during murine toxoplasmosis. Exp Parasitol 2012, 131:372-376.

33. Del RL, Butcher BA, Bennouna S, Hieny S, Sher A, Denkers EY: Toxoplasma gondii triggers myeloid differentiation factor 88-dependent IL-12 and chemokine ligand 2 (monocyte chemoattractant protein 1) responses using distinct parasite molecules and host receptors. J Immunol 2004, 172:6954-6960.

34. Hardison JL, Wrightsman RA, Carpenter PM, Kuziel WA, Lane TE, Manning JE: The CC chemokine receptor 5 is important in control of parasite replication and acute cardiac inflammation following infection with Trypanosoma cruzi. Infect Immun 2006, 74:135-143.

35. Gazzinelli RT, Ropert C, Campos MA: Role of the Toll/interleukin-1 receptor signaling pathway in host resistance and pathogenesis during infection with protozoan parasites. Immunol Rev 2004, 201:9-25.

36. Finkelman FD, Shea-Donohue T, Morris SC, Gildea L, Strait R, Madden KB, Schopf L, Urban JJ: Interleukin-4- and interleukin-13-mediated host protection against intestinal nematode parasites. Immunol Rev 2004, 201:139-155.

doi:10.1186/1756-3305-6-154

Cite this article as: Xu et al:: Characterization of mouse brain microRNAs after infection with cyst-forming Toxoplasma gondii. Parasites \& Vectors 2013 6:154.

\section{Submit your next manuscript to BioMed Central and take full advantage of:}

- Convenient online submission

- Thorough peer review

- No space constraints or color figure charges

- Immediate publication on acceptance

- Inclusion in PubMed, CAS, Scopus and Google Scholar

- Research which is freely available for redistribution

Submit your manuscript at www.biomedcentral.com/submit
Ciomed Central 